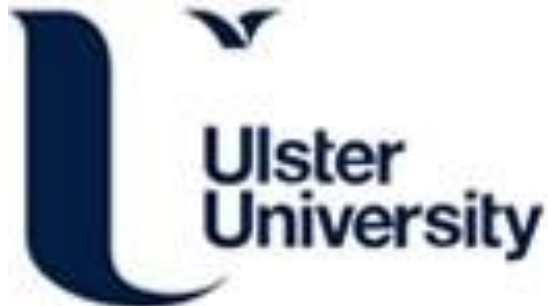

\section{UAV Position Estimation and Collision Avoidance using the Extended Kalman Filter}

\author{
Luo, C., McClean, S., Parr, G., Teacy, L., \& De Nardi, R. (2013). UAV Position Estimation and Collision \\ Avoidance using the Extended Kalman Filter. IEEE Transactions on Vehicular Technology, 62(6), 2749. \\ https://doi.org/10.1109/TVT.2013.2243480
}

Link to publication record in Ulster University Research Portal

\author{
Published in: \\ IEEE Transactions on Vehicular Technology
}

Publication Status:

Published (in print/issue): 01/01/2013

DOI:

10.1109/TVT.2013.2243480

\section{Document Version}

Author Accepted version

\section{General rights}

Copyright for the publications made accessible via Ulster University's Research Portal is retained by the author(s) and / or other copyright owners and it is a condition of accessing these publications that users recognise and abide by the legal requirements associated with these rights.

\section{Take down policy}

The Research Portal is Ulster University's institutional repository that provides access to Ulster's research outputs. Every effort has been made to ensure that content in the Research Portal does not infringe any person's rights, or applicable UK laws. If you discover content in the Research Portal that you believe breaches copyright or violates any law, please contact pure-support@ulster.ac.uk. 


\title{
UAV Position Estimation and Collision Avoidance using the Extended Kalman Filter
}

\author{
Chunbo Luo, Member, IEEE, Sally McClean, Member, IEEE, Gerard Parr, Member, IEEE, Luke Teacy, \\ and Renzo De Nardi
}

\begin{abstract}
Unmanned aerial vehicles (UAVs) play an invaluable role in information collection and data fusion. Because of their mobility and the complexity of deployed environments, constant position awareness and collision avoidance are essential. UAVs may encounter and/or cause danger if their Global Positioning System (GPS) signal is weak or unavailable. This paper tackles the problem of constant positioning and collision avoidance on UAVs in outdoor (wildness) search scenarios by using received signal strength (RSS) from the on-board communication module. Colored noise is found in the RSS, which invalidates the unbiased assumptions in Least Square (LS) algorithms which are widely used in RSS based position estimation. A colored noise model is thus proposed and applied in the extended Kalman filter for distance estimation. Furthermore, the constantly changing path loss factor during UAV flight can also affect the accuracy of estimation. In order to overcome this challenge, we present an adaptive algorithm to estimate the path loss factor. Given the position and velocity information, if a collision is detected we further employ an orthogonal rule to adapt the UAV predefined trajectory. Theoretical results prove that such an algorithm can provide effective modification to satisfy the required performance. Experiments have confirmed the advantages of the proposed algorithms.
\end{abstract}

Index Terms-Colored noise, position estimation, extended Kalman filter, collision avoidance

\section{INTRODUCTION}

The application of UAVs has attracted a lot of interest in research since the advancement of integrated circuit, artificial intelligence, robotics etc. [1]-[4]. It is thus possible that in the future a swarm of small but highly integrated UAV platforms will be able to work cooperatively and autonomously, especially in the time demanding scenarios. For example, in our Sensing Unmanned Autonomous Aerial VEhicles (SUAAVE) project funded by EPSRC, UK, the focus is on search and rescue, supported by reliable creation and control of swarms of UAVs in outdoor (wildness) scenarios [5]; however the work is more generally applicable to all autonomous control of UAVs. Such a deployment requires the capability of autonomous tasking and fast response.

Copyright (c) 2013 IEEE. Personal use of this material is permitted. However, permission to use this material for any other purposes must be obtained from the IEEE by sending a request to pubspermissions@ieee.org.

C. Luo, S. McClean and G. Parr are with the School of Computing and Information Engineering, University of Ulster, BT52 1SA UK e-mail: \{c.luo, si.mcclean, gp.parr\}@ulster.ac.uk.

L. Teacy is with the Electronics and Computer Science, University of Southampton, UK e-mail: wtlt@ecs.soton.ac.uk.

R. D. Nardi is with Department of Computing Science, University College London, UK e-mail: r.denardi@cs.ucl.ac.uk.
When a swarm of UAVs are deployed in outdoor scenarios, similarly to human controlled aerial vehicles, it is crucial to avoid potential collisions between each other as they fly fairly freely within the space. UAV formation and collision avoidance is a popular research topic, and some researchers have made significant improvements in this direction [1], [6]. However, it is still an emerging area which needs more theoretical contribution and practical experimentation. It is common to assume that the GPS signal is constantly available for each UAV during the lifetime of the task [6], however, such an assumption is not practical because the wireless satellite link may encounter physical blocks or interference. It has been found that even the placement of a GPS module given the weather condition can result in the reception of a weak signal or total failure [7], [8]. Moreover, some of the contributions are based on data from simulations rather than actual UAV experiments [6]. Also the results obtained might have bias towards practical UAV flights, e.g. the assumption of only white noise in radio frequency (RF) signal is not the whole story in RSS based position estimation.

The potential to use a wireless signal for the purpose of positioning has attracted great interest. Depending on the availability of measurements, three major wireless positioning methods were proposed in the literature ( [9]-[11]): 1) time of arrival (TOA) and/or time difference of arrival (TDOA); 2) angle of arrival (AOA), 3) received signal strength (RSS). The first two either require highly accurate equipment or need the support of antenna arrays, as well as a sophisticated signal processing module, which increases the payload, complexity and cost of UAV platform, even if they can be implemented on it. On the other hand, the RSS based method is a more convenient and economical solution if it is carefully handled, since the wireless communication equipment is already available on the UAV.

RSS based measurement has been studied in cellular networks and wireless local networks. The application of RSS in a cellular network was introduced in [12], which measures the forward control channels transmitted by the base station. The accuracy of using the RSS method is analyzed in [13], which shows that the RSS method is not as accurate as TDOA, but the accuracy provided is still useful to reduce the large errors that are sometimes introduced in TDOA and AOA based measurement. The lower bounds estimation when using RSS based maximum likelihood and linear kernel methods are given in [14]. The result reveals that in an urban area, the localisation error is higher when buildings are included in the urban scene 
as compared to a scenario where buildings are not present, which suggests the complexity and inconstancy of RSS. It is thus intuitive to use the Least Square (LS) algorithm to process the received RSS [13], [15], [16]. As evaluated in [17], the LS-based method is reasonably accurate if the radio propagation model is unbiased. However, due to correlated noise and the complexity of UAV flying attitude, such a requirement is rarely satisfied and the LS based methods could produce large-scale errors, which are also shown in the experiments (See Section VIII).

In order to improve the accuracy of the RSS based positioning algorithm, we use an extended Kalman filter (KF) [18] with colored noise model to estimate the position. Even though white noise is commonly assumed in RSS based algorithms [15], [19], from the results in [17] and our experiments analyzed by Allan variance [20], RSS not only has white noise but also correlated noise (See Section III). Many types of devices introduce correlated measurement errors, e.g. GPS receivers and the inertial measurement units (IMU) [21]. Similarly, in RSS based position estimation, if colored noise is not considered, the estimation has limited accuracy [13], [16]. Therefore in this paper, we proposed a colored noise model for the $\mathrm{KF}$ algorithm. Another factor that affects the estimation accuracy is the path loss factor in the propagation model. Researchers have found that, in real wireless channels, the path loss factor is not always constant $(\gamma \sim[1.6,6])$ [22]. We adapt this feature by estimating its value in real-time while the UAV flies. The probability of collision detection is then analyzed given the availability of position information. Of particular importance is the lower bound of collision probability and maximum number of repeated measurements, which are presented in this paper. We also propose to use the orthogonal rule for trajectory modification when a potential collision is detected and present the avoidance algorithm following this rule.

The contributions of this paper include:

1) Analysis and modelling of colored noise in the RSS measurements;

2) Improved estimation of the path loss factor in RF propagation model;

3) Adaptive safety zone modelling and design under imperfect position information;

4) Theoretical detection performance of the RSS based method and its practice;

5) The trajectory modification rule and its application.

The organization of this paper is as follows: Section II introduces the system modelling; Section III analyzes the noise in the radio measurements and models the colored noise; Section IV studies the position estimation algorithm; Section V proposes the estimation method of the path loss factor; Section VI gives the performance analysis of collision detection; Section VII proposes the collision avoidance rule and algorithm; Section VIII shows the experiment results; Section IX discusses the relevant issues and Section $\mathrm{X}$ concludes this paper.

Throughout this paper, ${ }^{\mathrm{T}}$ denotes transpose and $\log$ is the logarithm function with base $10 \cdot \min \{\cdot\}$ and $\max \{\cdot\}$ select

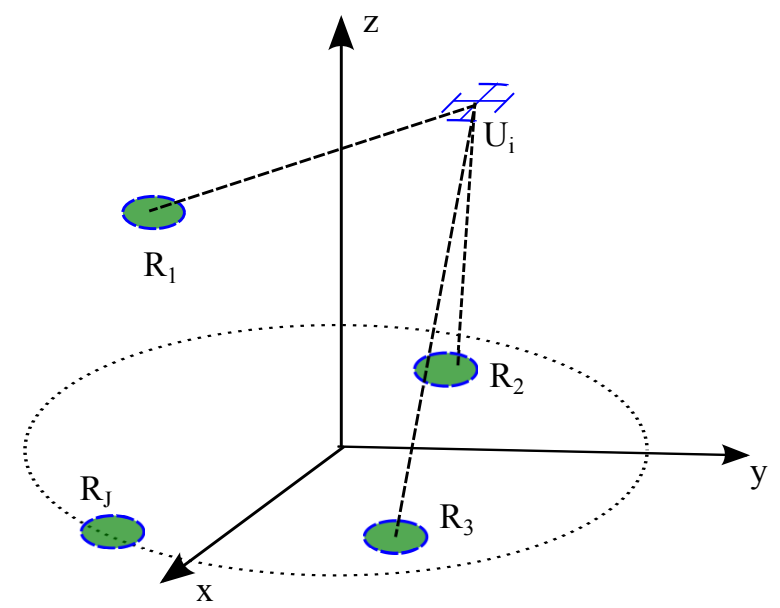

Fig. 1. The model of UAV position estimation. $R_{j}, j=1, \ldots, J$ are $\mathrm{J}$ reference nodes with known position information.

the minimum and maximum value of its input parameters respectively. $\|\cdot\|$ denotes the Euclidean norm, $\delta(\cdot)$ is the Dirac delta function and $\mathrm{E}(\cdot)$ calculates the expectation of its entry.

\section{System ModelLing}

\section{A. Position Estimation Model}

In order to know the position of an object, at least three reference nodes with known position information should be available. The model is shown in Fig. 1, where there are $J$ reference nodes, labelled as $R_{j}, j=1, \ldots, J$ and $I$ UAVs, $U_{i}, i=1, \ldots, I$, which need to estimate their own positions. The reference nodes can be other UAVs, local stations or access points which have accurate positions themselves and are synchronized to the global clock. This information can be obtained from the GPS onboard or off-line measurements and should be sufficiently accurate. Every UAV broadcasts a predefined control message at the given time stamp using wireless signals. These signals may suffer from fading, interference and other noise. Thus the received signal strength is normally affected by the deployment environment.

UAVs are modelled as rigid bodies and their positions are denoted as

$$
\mathbf{u}_{i}(n)=\left\{x_{i}(n), y_{i}(n), z_{i}(n)\right\}^{\mathrm{T}}, i=1, \ldots, I
$$

and the positions of reference nodes are given by

$$
\mathbf{r}_{j}(n)=\left\{x_{j}(n), y_{j}(n), z_{j}(n)\right\}^{\mathrm{T}}, j=1, \ldots, J
$$

where $n$ is the time stamp.

Within the given model, we can use three reference nodes to estimate the position of a UAV, given the availability of distance information. E.g. for $U_{i}$, the position equation is given below

$$
\left\|\mathbf{u}_{i}(n)-\mathbf{r}_{j}(n)\right\|=d_{i j}(n), j \in[1, \ldots, J], J \geq 3
$$

The key to employing this model is to estimate the distances between UAV and reference nodes using RSS provided by the onboard wireless communication module. 


\section{B. The Radio Communication Channel Model}

The wireless radio channel provides the essential medium for an electromagnetic wave to travel through, which suffers from extensive noise and interference. Furthermore, the movement of the transmitter and receiver bring a Doppler Effect and unstable attitude. Even though it is convenient to use the free-space model, this approach is not accurate in a real environment [22].

A more practical model studied in [22] provides a basic structure for the estimation of RSS in this paper. However, because of the complicated features of wireless propagation, the parameters of this model drift with changes in the physical environment. As a result we propose to use adaptive parameter estimation to formulize this practical problem.

The propagation model is given by [22]

$$
P_{r}=P_{t}+10 \log K-10 \gamma \log \frac{d_{i j}}{d_{0}}+v,
$$

where $P_{r}$ and $P_{t}$ are the received and transmitted power in $\mathrm{dBm} . K$ is a unitless constant that depends on the antenna characteristics and average channel attenuation, which can be determined by the measurement at $d_{0}$ [22]. In this paper, we set $d_{0}=1 m . d_{i j}$ is the distance between UAV $i$ and reference node $j . \gamma$ is the path loss factor. $v$ is the power of measurement noise in $\mathrm{dBm}$.

Set $d_{0}=1 \mathrm{~m}$ and denote the measurement at the time stamp $n$ as $\varphi(n)=P_{r}(n)-P_{t}(n)-10 \log K$, the path loss model can be simplified as follows

$$
\varphi(n)=-10 \gamma(n) \log d_{i j}(n)+v(n) .
$$

This model will be used to estimate the distance.

It is usually assumed in the literatures, e.g. [19], [22], that the noise in (3) is Gaussian distributed. However, based on the data collected from our extensive UAV experiments and [17], colored noise plays a significant role in the practical scenarios, which will be discussed in the next section.

\section{Noise ANAlysis AND Modelling}

\section{A. Noise Analysis}

A segment of radio frequency signal received by the UAV is shown in Fig.2, which is similar to the recordings in [19]. It is interesting to notice that, besides white noise, the radio signal also shows the impact of colored noise (Fig.3), which is analyzed by the tool of Allan variance [23]. Allan variance is based on cluster analysis [24] and is a powerful tool to disguise the overall noise statistics.

Denote the correlation time as $\tau$ and the sampling frequency as $f$ and suppose we have a collection of $N$ data $\varphi(n), n=1, \ldots, N$, the correlation can be revealed by grouping these data into $M=\frac{N}{f}$ clusters and computing the variance as follows

$$
\sigma_{\tau}^{2}=\frac{1}{2}\left\|\mu_{k+1}(M)-\mu_{k}(M)\right\|^{2}, k=1, \ldots, f-1
$$

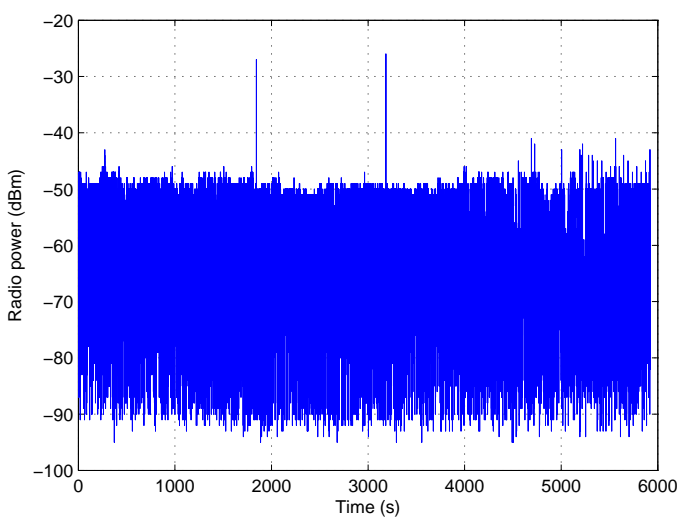

Fig. 2. One segment of the received radio signal.

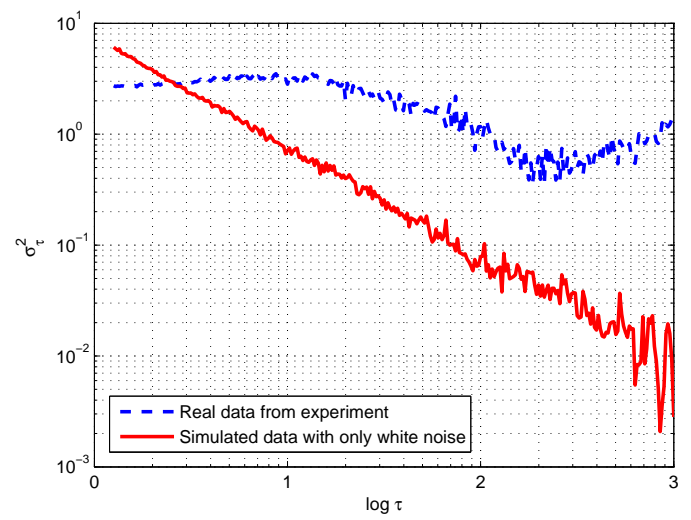

Fig. 3. Allan variance.

where

$$
\mu_{k}(M)=\frac{1}{M} \sum_{i=(k-1) M+1}^{k M} \varphi(i), k=1, \ldots, f-1
$$

Allan variance of the received radio signal is shown in Fig. 3. In the figure, the Allan variance of simulated data with only white noise is also shown. From the slope of $\log \log$ spectrum, we can see the slope of the simulated data is -1 and the real radio data is severely affected by colored noise, especially at the short correlation time area (e.g. $\log \tau<1$ ) where the slope of correlation in real data is positive. when $\log \tau$ is above 2 , the contribution from correlated noise can also be observed.

The error of root Allan variance, $\sigma_{\tau}$, decreases with the increase of frequency $f$, as shown in Fig.3. The chi-squared distribution is commonly used to establish its confidence interval, expressed as

$$
\chi^{2}=\frac{\digamma \sigma_{\tau}^{2}}{\sigma^{2}},
$$

where $\sigma^{2}$ is the true variance value and $\digamma$ is the degree of freedom for the estimator. $\chi^{2}$ denotes the cumulative distribution function of the chi-squared distribution. Given the confidence of $\varepsilon$, the confidence of Allan variance 
estimation is given by

$$
\frac{\digamma \sigma_{\tau}^{2}}{\chi^{2}(\varepsilon)} \leq \sigma^{2} \leq \frac{\digamma \sigma_{\tau}^{2}}{\chi^{2}(1-\varepsilon)}
$$

\section{B. Colored Noise Modelling}

In the case of correlated noise observation, such as the radio signals in Section III, we can use a Gaussian Markov process (GMP) to model the correlation [25], [26], generated by a continuous time autoregressive model given below

$$
\dot{\phi}(t)=\lambda \phi(t)+w_{2}(t),
$$

where $\lambda$ is the coefficient of GMP and $w$ is additive white noise $w_{2} \sim N\left(0, \sigma_{w_{2}}^{2}\right)$ and $E\left[w_{2}(t) w_{2}(t-T)\right]=\delta(T)$, $\delta(\cdot)$ is the Dirac delta function.

Firstly (7) is converted to the discrete time domain

$$
\phi(n)=e^{\lambda \Delta} \phi(n-1)+e^{\lambda \Delta} w_{2}(n),
$$

where $\Delta$ denotes the time incremental.

Driven by the exciting noise $w_{2}(n)$, the correlation between noises $\phi(m)$ and $\phi(n)$ is given by

$$
\begin{aligned}
R_{\phi}(m, n)= & E[\phi(m) \phi(n)] \\
= & E\left[\left(e^{\lambda \Delta m} \phi(0)+\sum_{i=1}^{m} e^{\lambda \Delta i} w_{2}(m-i+1)\right)\right. \\
& \left.\left(e^{\lambda \Delta n} \phi(0)+\sum_{j=1}^{n} e^{\lambda \Delta j} w_{2}(n-j+1)\right)\right] \\
= & e^{\lambda \Delta(m+n)} E\left[\phi^{2}(0)\right]+\sum_{i=1}^{m} \sum_{j=1}^{n} e^{\lambda \Delta(i+j)} \\
& E\left[w_{2}(m-i+1) w_{2}(n-j+1)\right] .
\end{aligned}
$$

Without losing generality, we assume $n \geq m$. The correlation can be denoted as

$$
\begin{aligned}
R_{\phi}(m, n) & =e^{\lambda \Delta(m+n)} E\left[\phi^{2}(0)\right] \\
& +\sigma_{w_{2}}^{2} e^{\lambda \Delta(n-m)} \sum_{i=1}^{m} e^{\lambda \Delta(2 i)} .
\end{aligned}
$$

From (10), it is easy to see that, in order to maintain the convergence of the model, $\lambda$ must be less than 0 . When $m$ and $n$ are large, the correlation can be approximated as

$$
R_{\phi}(m, n) \approx \frac{\sigma_{w_{2}}^{2} e^{\lambda \Delta(n-m+2)}}{1-e^{2 \lambda \Delta}} .
$$

By using the measured data, its Allan variance and (11), the three parameters $\lambda, \Delta$ and $\sigma_{w_{2}}^{2}$ can be obtained. For example, the data shown in Fig.2 were measured under the time increment of $0.1 s$, thus $\Delta=0.1 s$. Two points can be selected from Fig.3 to calculate the two unknown parameters, where $\tau=\Delta(n-m)$.

\section{Position EStimation}

In this section, we introduce an algorithm to estimate distance using RSS. With the distance information between
UAVs and the reference sites, its position can be calculated straightforwardly using (1).

Based on the analysis introduced in previous sections, the distance between the $i$ th UAV and $j$ th reference node can be modelled as (The subscript $i j$ is neglected to simplify the expression in the rest of this paper.)

$$
\left\{\begin{array}{l}
d(n)=d(n-1)+\psi(n-1)+w(n-1), \\
\varphi(n)=10 \gamma(n) \log d(n)+\phi(n)+v(n),
\end{array}\right.
$$

where $\psi(n-1)$ is the optional control input, e.g. IMU measurements, and $w(n-1)$ is the estimation noise, which is often modelled as Gaussian distributed, e.g. $N\left(0, \sigma^{2}\right)$, $\phi(n)$ is the colored measurement noise and $v(n)$ is white measurement noise with variance $\mathbf{R}$. The initial conditions at $n=0$ are given as $d(0)=d_{0}, \psi(0)=0$.

We apply the extended Kalman Filter to estimate the distance $d(n)$. The first equation in (12) denotes the state and the second one is the measurement equation. Using the colored noise model of (7), we can write the colored noise state as follows

$$
\phi(n)=e^{\lambda \Delta} \phi(n-1)+e^{\lambda \Delta} w_{2}(n-1),
$$

where $w_{2}(n-1)$ is the white noise in (7) with variance $\sigma_{w}^{2}, \lambda$ and $\Delta$ are also given by (7).

The two state equations - (13) and the first equation in (12) - are combined together using the following expression

$$
\mathbf{c}(n)=f(\mathbf{c}(n-1), \boldsymbol{\psi}(n-1), \mathbf{w}(n-1)),
$$

where $\mathbf{c}(n-1)=[d(n-1), \phi(n-1)]^{\mathrm{T}}, \boldsymbol{\psi}(n-1)=$ $[\psi(n-1), 0]^{\mathrm{T}}$ and $\mathbf{w}(n-1)=\left[w(n-1), w_{2}(n-1)\right]^{\mathrm{T}}$. The process noise matrix is given by $\mathbf{Q}=\mathrm{E}\left\{\mathbf{w w}^{\mathrm{H}}\right\}$, and the Jacobian matrices of $f(\cdot)$ are calculated as

$$
[\mathbf{A}(n)]_{i j}=\frac{\partial f_{i}}{\partial c_{j}},[\mathbf{W}(n)]_{i j}=\frac{\partial f_{i}}{\partial w_{j}}, i, j=1,2 .
$$

The measurement equation for the Kalman filter is given as follows

$$
\varphi(n)=h(\mathbf{c}(n))+v(n),
$$

where $h(\mathbf{c}(n))=10 \gamma(n) \log c_{1}(n)+\phi(n)$. The partial derivatives of (15) generate its Jacobian matrix

$$
[\mathbf{h}(n)]_{j}=\frac{\partial h}{\partial c_{j}}, j=1,2 .
$$

Combing (14) and (15) gives us the essential components of the Kalman filter algorithm. The prediction of state vector is then given by

$$
\tilde{\mathbf{c}}(n)=f(\hat{\mathbf{c}}(n-1), \boldsymbol{\psi}(n-1), 0)) .
$$

The a priori estimate error covariance, $\tilde{\mathbf{P}}(n)$, is calculated as

$$
\tilde{\mathbf{P}}(n)=\mathbf{A}(\tilde{\mathbf{c}}(n)) \mathbf{P}(n-1) \mathbf{A}^{\mathrm{T}}(\tilde{\mathbf{c}}(n))+\mathbf{W}(n) \mathbf{Q} \mathbf{W}^{\mathrm{T}}(n),
$$

where $\mathbf{P}(n-1)$ is the a posteriori estimate error covariance. Its initial condition is given empirically as $\mathbf{P}(0)=1$.

The measurement sensitivity would affect the error covariance, given by

$$
\hat{\mathbf{P}}(n)=\tilde{\mathbf{P}}(n) \mathbf{h}^{\mathrm{T}}(n) .
$$


Now we can update the Kalman gain as follows

$$
\mathbf{K}(n)=\hat{\mathbf{P}}(n)(\mathbf{h}(n) \hat{\mathbf{P}}(n)+\mathbf{R})^{-1} .
$$

The estimation of state $\hat{\mathbf{c}}(n)$ is thus calculated as

$$
\hat{\mathbf{c}}(n)=\tilde{\mathbf{c}}(n)+\mathbf{K}(n)(\varphi(n)-h(\tilde{\mathbf{c}}(n))) .
$$

The a posteriori estimate error covariance is finally updated by

$$
\mathbf{P}(n)=(\mathbf{I}-\mathbf{K}(n) \mathbf{h}(n)) \tilde{\mathbf{P}}(n) .
$$

Performance of the algorithm will be verified in Section VIII, which shows that $80 \%$ of estimations have estimation error less than $4 \mathrm{~m}$. The distance obtained is directly used in (1) for position estimation, which is neglected here.

\section{Estimation of Path Loss Factor $\gamma$}

UAVs are always moving and, as a result, the common assumption of fixed path loss factor of the radio model is not practical since the environment is constantly changing. In this paper, we use a modified Least Square (LS) method to estimate the path loss factor. The path loss model (3) is described as follows

$$
\varphi(n)=-10 \gamma(n) \log d_{i j}(n)+\phi(n)+v(n) .
$$

Denote $f(\gamma(n))=-10 \gamma(n) \log d_{i j}(n)$ and we assume there are $M$ previous data available. The error functions can be written as

$$
\left\{\begin{array}{l}
e(n)=\varphi(n)-f(\gamma(n)) \\
e(n-1)=\varphi(n-1)-f(\gamma(n-1)) \\
\cdots \\
e(n-M+1)=\varphi(n-M+1)-f(\gamma(n-M+1))
\end{array}\right.
$$

Stacking them into vectors: $\mathbf{e}(n)=[e(n), e(n-$ $1), \ldots, e(n-M+1)]^{\mathrm{T}}, \mathbf{L}(n)=[\varphi(n), \varphi(n-1), \ldots, \varphi(n-$ $M+1)]^{\mathrm{T}}$ and $\mathbf{F}(n)=[f(\gamma(n)), f(\gamma(n-1)), \ldots, f(\gamma(n-$ $M+1))]^{\mathrm{T}}$,

$$
\begin{aligned}
\varepsilon(\gamma(n)) & =\mathbf{e}(n)^{\mathrm{T}} \mathbf{e}(n) \\
& =\mathbf{F}^{\mathrm{T}}(n) \mathbf{F}(n)-2 \mathbf{F}^{\mathrm{T}}(n) \mathbf{L}(n)+\mathbf{L}^{\mathrm{T}}(n) \mathbf{L}(n) .
\end{aligned}
$$

Since $\mathbf{L}^{\mathrm{T}}(n) \mathbf{L}(n)$ is a constant, to have the minimum value of $\varepsilon(\gamma(n))$ leads to

$$
\hat{\varepsilon}(\gamma(n))=\min \left\{\mathbf{F}^{\mathrm{T}}(n) \mathbf{F}(n)-2 \mathbf{F}^{\mathrm{T}}(n) \mathbf{L}(n)\right\} .
$$

We cannot have an explicit expression of $\gamma$ from its first order derivative because $\mathbf{F}(n)$ includes series of non-linear functions with unknown parameters. Thus the linear method to obtain the ideal $\gamma$ by assigning 0 to its first order derivative cannot work here.

Instead, an improved Gauss-Newton algorithm is presented in this paper to estimate $\gamma$ at the $n$th time stamp by iterations. Based on the LS theory, estimation of $\gamma$ can be given by

$$
\gamma^{(s+1)}=\gamma^{(s)}+\theta \Theta^{(s)},
$$

where $\Theta^{(s)}=\mathbf{J}^{\mathrm{T}}\left(\gamma^{(s)}\right) \mathbf{J}^{-1}\left(\gamma^{(s)}\right) \mathbf{J}^{\mathrm{T}}\left(\gamma^{(s)}\right) \mathbf{e}(n)$ and $\theta$ is the adjustment factor employed to improve performance as analyzed below. The Jacobian matrix $\mathbf{J}(\gamma)=$ $\left[\frac{\partial e_{1}(n)}{\partial \gamma}, \ldots, \frac{\partial e_{N-M+1}(n)}{\partial \gamma}\right]^{\mathrm{T}}$ is calculated and used for iterations. $s$ is the iterative index. Because Gauss-Newton method heavily depends on the initial value and may diverge, we employ the small parameter $\theta$ to improve the performance.

Firstly we prove the necessity of introducing $\theta$. It is easy to know that $\varepsilon^{\mathrm{T}}\left(\gamma^{(s)}\right) \varepsilon\left(\gamma^{(s)}\right)$ is not minimum because $\gamma^{(s)}$ is only an approximation to the true value of $\gamma$. Then we have

Theorem 1: Since

$$
\left.\frac{\partial \varepsilon^{\mathrm{T}}(\gamma) \varepsilon(\gamma)}{\partial \gamma}\right|_{\gamma=\gamma^{(s)}} \neq 0,
$$

a positive $\Lambda$ for $\theta \in[0, \Lambda]$ must exist so that

$$
\varepsilon^{\mathrm{T}}\left(\gamma^{(s)}+\theta \Theta^{(s)}\right) \varepsilon\left(\gamma^{(s)}+\theta \Theta^{(s)}\right)<\varepsilon^{\mathrm{T}}\left(\gamma^{(s)}\right) \varepsilon\left(\gamma^{(s)}\right),
$$

where $\Theta^{(s)}=\left[\mathbf{J}^{\mathrm{T}}(\gamma) \mathbf{J}(\gamma)\right]^{-1} \mathbf{J}^{\mathrm{T}}(\gamma) \mathbf{e}(n)$,

Proof: See appendix.

The choice of $\theta$ is based on the scenarios, however, it is set to 0.5 for simplicity in our experiments. Even though this value is probably not optimal, it is expected to work properly also in other cases. To find its optimal value is an interesting topic for further research. The stop condition is given by

$$
\left|\varepsilon\left(\gamma^{(s)}\right)-\varepsilon\left(\gamma^{(s-1)}\right)\right| \leq \mu
$$

where $\mu$ is a predefined error tolerance.

The algorithm for the computation of $\gamma(n)$ is summarized below,

1) For a given initial value $\gamma^{(0)}$ and $\Theta^{(0)}$ are computed.

2) $\gamma^{(s)}$ and $\varepsilon\left(\gamma^{(s)}\right)$ are calculated based on (25) and (23).

3 ) If the stop condition (28) is satisfied, go to step 4). Otherwise go to step 2).

4) $\gamma^{(s)}$ is output to $\gamma(n)$.

\section{COLlision Detection Under IMPERFECT DISTANCE ESTIMATION}

In this paper, we set a safety zone for each UAV, as shown in Fig.4. A collision warning will be generated if the safety zone is violated by another $\operatorname{UAV} u_{k}(k \neq i)$,

$$
\begin{cases}d_{i, k} \leq S_{i, k}, & \text { Warning, } \\ d_{i, k}>S_{i, k}, & \text { Safe. }\end{cases}
$$

The distance $d_{i, k}$ can be directly calculated from RSS measurements.

Manned aerial vehicles usually maintain a fixed safety zone [27]. Perfect detection can be achieved if we have accurate information of the distance between each pair of UAVs. In this case, the radius of the safety zone can be set as follows

$$
S_{i, k}=\left\|\hat{\mathbf{v}}_{i, k} * t_{r}\right\|, \text { Perfect condition, }
$$

where $\hat{\mathbf{v}}_{i, k}$ is the maximum relative velocity between the two UAVs and $t_{r}$ is the reaction time. $\hat{\mathbf{v}}_{i, k}$ can be set by a default value or can be calculated from the communications between the two UAVs. 


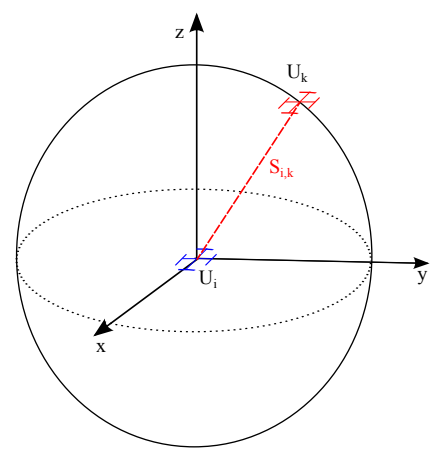

Fig. 4. The UAV safety zone. $S_{i, k}$ is the radius of $U_{i}$ 's safety zone.

However, because the RSS based distance estimation has limited accuracy, we have to study the collision detection under imperfect distance estimation. Intuitively, if one experiment has a certain level of accuracy, we can carry out multiple independent experiments to improve it.

Firstly we define $P\left(\hat{\delta}_{d}\right)$ as the probability of one estimation error less than or equal to $\hat{\delta}_{d}$

$$
P\left(\hat{\delta}_{d}\right)=P\left(\text { one measurement error } \leq \hat{\delta}_{d}\right)
$$

Then the probability of one estimation error greater than $\hat{\delta}_{d}$ should be $1-P\left(\hat{\delta}_{d}\right)$. Given $n_{d}$ independent estimations, the probability that all of them have errors greater than $\hat{\delta}_{d}$ is as follows

$$
P\left(n_{d} \text { measurements errors }>\hat{\delta}_{d}\right)=\left(1-P\left(\hat{\delta}_{d}\right)\right)^{n_{d}} .
$$

The values of $\hat{\delta}_{d}$ and $P\left(\hat{\delta}_{d}\right)$ can be determined as follows

$$
P\left(\hat{\delta}_{d}\right)=\int_{0}^{\hat{\delta}_{d}} p\left(\delta_{d}\right) d \delta_{d}
$$

where $p\left(\delta_{d}\right)$ is PDF of the errors, $\delta_{d}$. For example, in the experiments we found that $80 \%$ of estimation errors are within $4 \mathrm{~m}$, thus $\hat{\delta}_{d}=4 \mathrm{~m}, P\left(\hat{\delta}_{d}\right)=80 \%$.

Furthermore, it is easy to have the following inequality:

$$
\begin{gathered}
\text { Probability }\left(\text { Real distance of }(\mathrm{i}, \mathrm{k}) \leq S_{i, k} \mid d_{i, k}>S_{i, k}\right) \\
\leq P\left(\text { one measurement error }>\hat{\delta}_{d}\right)
\end{gathered}
$$

since the two UAVs' safety zones may not overlap even if the estimation error is greater than the threshold.

Let $\mathbb{P}$ be the collision probability. We assume that if the distance measurement causes a warning, a proper control action will be available to avoid a possible collision (such action is introduced in Section VII). Therefore

$$
\begin{gathered}
\mathbb{P} \leq \text { Probability }\left(\text { Real distance of }(\mathrm{i}, \mathrm{k}) \leq S_{i, k} \mid\right. \\
\left.\quad d_{i, k}>S_{i, k},\{i, k\} \subset\{1, \ldots, I\}\right)
\end{gathered}
$$

for the following reason: even if a collision always occurs when Real distance of $(i, k) \leq S_{i, k}$, the collision probability is obtained from the right side of the above equation by multiplying it with the probability of having two UAVs within each other's safety zone.

In the case of distance errors lower than $\hat{\delta}_{d}$, the warning rules and control actions are assumed to be able to compen- sate for the distance errors and avoid collision. According to the analysis above, a collision can occur only when all the $n_{d}$ independent measurement errors are larger than $\hat{\delta}_{d}$, e.g.

$$
\mathbb{P} \leq\left(1-P\left(\hat{\delta}_{d}\right)\right)^{n_{d}} .
$$

From the above equation, we can obtain the minimum value of $n_{d}$ to ensure that $\mathbb{P} \leq\left(1-P\left(\hat{\delta}_{d}\right)\right)^{n_{d}}<\overline{\mathbb{P}}$, where $\overline{\mathbb{P}}$ is the desired maximum collision probability.

Given the distribution of errors, $P\left(\hat{\delta}_{d}\right)$, and the desired maximum collision probability, $\overline{\mathbb{P}}$, the number of independent experiments can be calculated as follows,

$$
n_{d}=\frac{\log \overline{\mathbb{P}}}{\log \left(1-P\left(\hat{\delta}_{d}\right)\right)},
$$

where $n_{d}$ is rounded to the nearest integer towards infinity. Given the example of $P(4)=80 \%, \overline{\mathbb{P}}=10^{-5}$, we can obtain $n_{d}=8$. In other words, the collision probability from RSS measurements can be limited to $10^{-5}$ if 8 independent trials are taken for the same channel between the two UAVs.

Finally, to ensure that the measurements are independent, the measurement frequency has to be lower than the value imposed by the channel coherence. This corresponds to a constraint on the maximum value of $n_{d}$ and the minimum collision probability that the system can reach.

Proposition 1: In the RSS based methods, the collision probability corresponding to imperfect RSS measurement is lower bounded by

$$
\mathbb{P}\left(\hat{\delta}_{d}\right)=\left(1-P\left(\hat{\delta}_{d}\right)\right)^{N_{d}}
$$

given the distribution of error in estimation (See Fig.5) and $N_{d}=\left\lfloor 1 / T_{d}\right\rfloor, T_{d}$ is the channel coherence time and $\lfloor\cdot\rfloor$ selects the largest integer not greater than its input parameter. The maximum number of independent measurements is $N_{d}$.

Proof: From the CDF of errors in the distance estimation shown in Fig.5, the following relationship can be obtained, $P\left(\hat{\delta}_{d}\right)=\alpha \%$, where $\hat{\delta}_{d}$ is the estimation error, which means $\alpha \%$ of the estimations have errors within the range $\left[0, \hat{\delta}_{d}\right]$. The probability of estimation error greater than $\hat{\delta}_{d}$ is given by

$$
P^{\prime}\left(\hat{\delta}_{d}\right)=1-P\left(\hat{\delta}_{d}\right)=1-\alpha \%
$$

If the frequency of estimation is $n_{d}$ and the error probability distribution function (PDF) of $n_{d}$ estimations is $p^{\prime}\left(\delta_{d_{1}}, \ldots, \delta_{d_{n_{d}}}\right)$, the CDF of false detection can be calculated as

$$
\mathbb{P}\left(\delta_{d_{1}}, \ldots, \delta_{d_{n_{d}}}\right)=\int \ldots \int p^{\prime}\left(\delta_{d_{1}}, \ldots, \delta_{d_{n_{d}}}\right) d \delta_{d_{1}} \ldots d \delta_{d_{n_{d}}} .
$$

The right-hand-side of the above equation is bounded by the following special case: each estimation is independent 


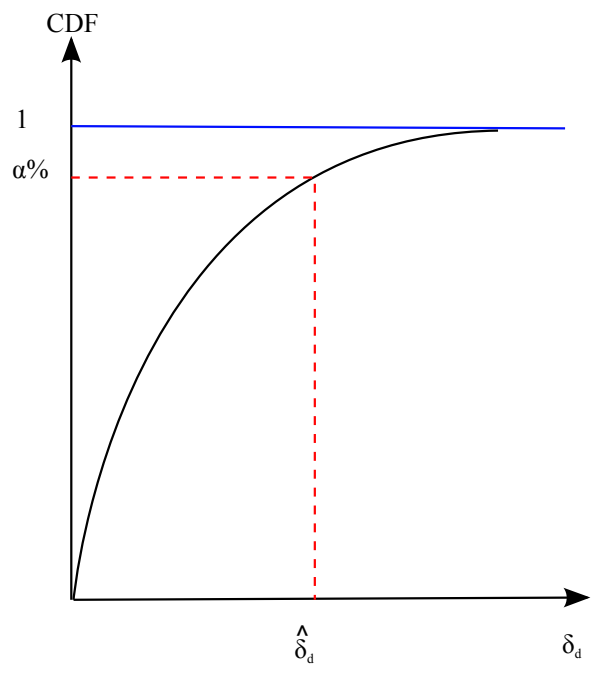

Error in distance estimation $(\mathrm{m})$

Fig. 5. $\mathrm{CDF}$ of error in distance estimation. $\alpha \%$ of the errors are not greater than $\hat{\delta_{d}} m$.

of the others:

$$
\begin{aligned}
\int \ldots \int p^{\prime}\left(\delta_{d_{1}}, \ldots, \delta_{d_{n_{d}}}\right) d \delta_{d_{1}} \ldots d \delta_{d_{n_{d}}} \\
\quad \geq \int p^{\prime}\left(\delta_{d_{1}}\right) d \delta_{d_{1}} \ldots \int p^{\prime}\left(\delta_{d_{n_{d}}}\right) d \delta_{d_{n_{d}}} \\
\quad=p^{\prime}\left(\delta_{d_{1}}\right) \cdots p^{\prime}\left(\delta_{d_{n_{d}}}\right) .
\end{aligned}
$$

Since the RSS based method is employed in this paper, the independence of a group of continuous estimates is imposed by the channel coherence time $T_{d}$. As a result, the maximum estimation frequency is

$$
N_{d}=\left\lfloor 1 / T_{d}\right\rfloor .
$$

If the same error threshold $\hat{\delta}_{d}$ is given to every single estimation, e.g. $\delta_{d_{i}}, i=1, \ldots, n_{d}$, (38) is proved.

For example, if an IEEE 802.11 wireless signal is employed for position estimation, at a mobility of $1 \mathrm{~m} / \mathrm{s}(3.6 \mathrm{~km} / \mathrm{h})$, the coherence time is approximately $25.39 \mathrm{~ms}$, and this value reduces to $12.69 \mathrm{~ms}$, and $8.46 \mathrm{~ms}$ for the speed of $2 \mathrm{~m} / \mathrm{s}(7.2 \mathrm{~km} / \mathrm{h})$, and $3 \mathrm{~m} / \mathrm{s}(10.8 \mathrm{~km} / \mathrm{h})$ respectively [28]. Higher speed leads to even lower coherence time. If we choose $T_{d}=25.39 \mathrm{~ms}$, the maximum number of independent measurements can be obtained as

$$
N_{d}=\frac{1}{T_{d}} \geq \frac{1}{25.39 * 10^{-3}} \approx 40 .
$$

Under the condition of imperfect distance information, the safety zone has to be set carefully. If it is too large, the deployment and cooperation of UAVs would be greatly restricted as each UAV has to keep a fairly large distance from the others; if it is too small, once a potential collision is detected, the time left for collision avoidance may not be enough. Thus the safety zone for UAV under imperfect distance information can be given as

$S_{i, k}=\left(\frac{\hat{\delta}_{d}}{P\left(\hat{\delta}_{d}\right)}+\left\|\hat{\mathbf{v}}_{i, k} * t_{r}\right\|\right) * n_{d}$, Imperfect conditions.

For example, if $\hat{\delta}_{d}=4 m, P\left(\hat{\delta}_{d}\right)=80 \%, \hat{\mathbf{v}}=$ $\{10,0,0\} \mathrm{m} / \mathrm{s}, t_{r}=1 \mathrm{~s}, n_{d}=8$, the safety zone radius $S=120 m$ as shown in Fig. 6 .

\section{COllision Avoidance}

In this section, we study the control rules if two UAVs have potential collision. The avoidance action can be formulated as the regulator problem [29], [30]. Given the dynamical system of UAVs as

$$
\mathbf{u}_{i}(n+1)=\mathbf{u}_{i}(n)+\mathbf{v}_{i}(n),
$$

where $\mathbf{v}_{i}(n)$ is the velocity per frame. The following cost function can be defined,

$J_{i}(n)=q(n)\left\|\mathbf{u}_{i}(n)-\mathbf{u}_{i}^{d}(n)\right\|+\kappa(n)\left\|\mathbf{v}_{i}(n)-\mathbf{v}_{i}(n-1)\right\|$,

where $\mathbf{u}_{i}^{d}(n)$ is the predefined trajectory, which will be mandatorily modified if a potential collision is detected. The model predictive controller proposed in [29] is used to adjust the movement of $U_{i}$, which is neglected here. $q(n)$ and $\kappa(n)$ are the weight factors for tracking error and velocity increment respectively.

The cooperative collision avoidance scheme works as follows. We assume every UAV has the same size of safety zone and a unique identity number (ID). However, different a safety zone can be easily set where the safety zone with smaller size should be considered. Without losing generality, the UAVs with lower ID are given higher priority. Once a potential collision is detected (29), the two involved UAVs $\left(U_{i}, U_{j}, i<j\right)$ will exchange information including $I D$, velocity $\mathbf{v}$ and current and the next way point $\mathbf{u}$. The collision avoidance algorithm will then work by the following steps

- If

$$
\left\|\mathbf{u}_{i}(n+1)-\mathbf{u}_{j}(n+1)\right\| \geq S_{i, j},
$$

at time $n+1$ the safety zones of the two UAVs are disjoint so no action is needed: $\mathbf{u}_{i}(n+1)=\mathbf{u}_{i}^{d}(n+$ $1), \mathbf{u}_{j}(n+1)=\mathbf{u}_{j}^{d}(n+1)$.

- If

$$
\left\|\mathbf{u}_{i}(n+1)-\mathbf{u}_{j}(n+1)\right\|<S_{i, j},
$$

$U_{j}$ 's trajectory must be modified following the orthogonal rule introduced below.

The Orthogonal Rule: When two UAVs $\left(U_{i}, U_{j}, i<\right.$ $j$ ) have potential collision, a new way point of the UAV with greater ID, $\mathbf{u}_{j}(n+1)$, should be on the edge of $U_{i}$ 's safety zone, the direction of way point change should be vertical to the velocity of $U_{i}$, and the value of way point change should be the smallest.

The advantage of this rule is to ensure collision avoidance with acceptable complexity and low implementation difficulty. The chosen way points can be connected using 


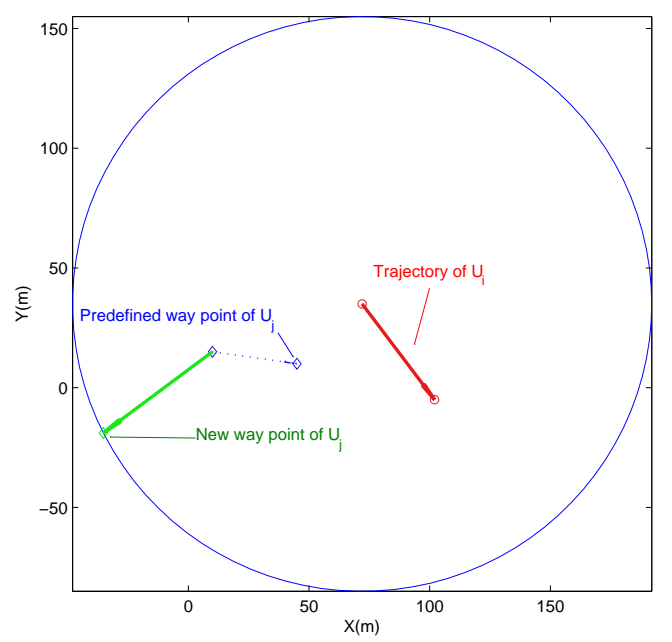

Fig. 6. UAV trajectory adjustment. Blue circle is the edge of $U_{i}$ 's safety zone. $U_{i}$ follows its old trajecotory while $U_{j}, j>i$ modifies its trajectory if a potential collision is detected.

more sophisticated algorithms. For example, genetic algorithms and higher order polynomial approximation can be used to find a smooth trajectory between two way points. Particularly when vehicles are at high velocities, it is better for them to follow a curved trajectories.

Following the orthogonal rule, the next way point of $U_{j}$, $\mathbf{u}_{j}(n+1)$, can be calculated as follows,

$$
\left\{\begin{array}{l}
\left\|\mathbf{u}_{j}^{\prime}(n+1)-\mathbf{u}_{i}(n)\right\|=S_{j, i}, \\
{\left[\mathbf{u}_{j}^{\prime}(n+1)-\mathbf{u}_{j}(n)\right] \cdot \mathbf{v}_{i}(n)=0,} \\
\mathbf{u}_{j}(n+1)=\min _{\mathbf{u}_{j}^{\prime}(n+1)}\left\|\mathbf{u}_{j}^{\prime}(n+1)-\mathbf{u}_{j}(n)\right\| .
\end{array}\right.
$$

The change of trajectory is illustrated in Fig. 6 (In 2 Dimention). More experimental results are given in Section VIII. Once the danger is eliminated, they will follow their own predefined trajectories again.

\section{EXPERIMENTS}

\section{A. Dataset preparation}

The experiments were divided into two steps. Firstly, the dataset was collected and prepared. Then the data was post processed by LS and Kalman filters. The dataset was acquired in practical experiments by quadrotor UAVs (Fig. 7) in University College London. On the UAV platform, there is a GPS receiver (Ublox LEA4T) to record position information, which was post processed using reference information received by the GPS base station in University College London. With the usage of highly sensitive antennas and accurate geographical information of the base station, the error between GPS measurements and UAV's true position is at the level of $10 \mathrm{~cm}$, which is also confirmed by manual measurements. Three gyros (Analog Devices ADXRS610), a tri-axial accelerometer (Memsic MXR9500), three magnetometers (NXP KMZ51) and a pressure sensor (Freescale MPXH6115) are fused at the rate

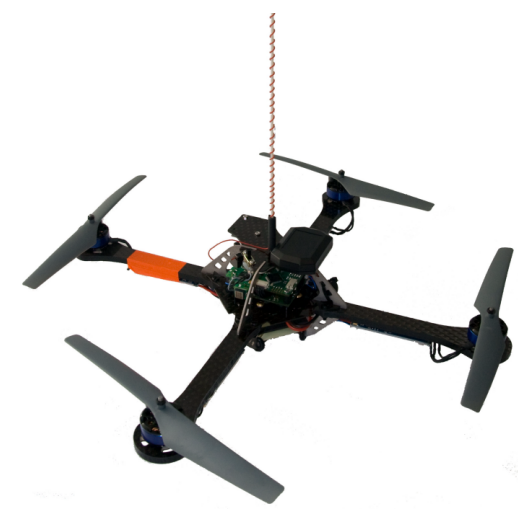

Fig. 7. AscTec hummingbird quadrocopter.

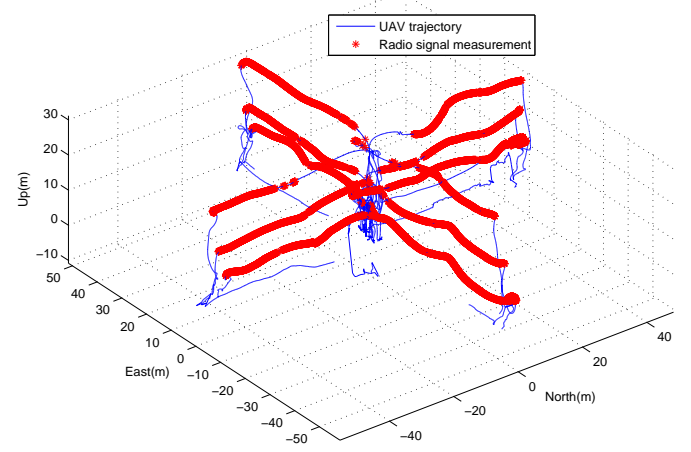

Fig. 8. This figure shows the flight trajectory of the UAV in experiments (blue line) and the locations where radio measurements were recorded (red asterisks).

of $1 \mathrm{KHz}$ by the on-board firmware of UAVs to provide the IMU data.

The radio frequency signal used in our experiments was from IEEE 802.11 transceivers. We used two mini PCI express $802.11 \mathrm{~b} / \mathrm{g}$ modules (Gigabyte GN-WI01GT) on both the transmitter and receiver to provide the required signal. These 802.11 modules were set at the fixed channel of $2.462 \mathrm{GHz}$, which was tested to be free from other transmissions. The transmission power was $5 \mathrm{dBm}$ and we set a fixed transmission rate of $1 \mathrm{Mb} / \mathrm{s}$.

Trajectories of UAVs and the locations where RSS were recorded are shown in Fig.8. The positions are expressed in a local coordinate frame centered at the ground antenna location, hence the altitude can be negative, and aligned to the main flight directions. Flights were carried out in an open outdoor area at varied velocity between $0 \mathrm{~m} / \mathrm{s}$ and about $5 \mathrm{~m} / \mathrm{s}$. For each of the four main directions (North, South, East, West) and for each one of three flight altitudes $(10 \mathrm{~m}, 20 \mathrm{~m}, 30 \mathrm{~m})$, we conducted a total of 12 distinct flights.

The collected data will be analyzed by the proposed algorithm and the widely used LS method [14], [15]. Unless specifically mentioned, both the two will use the same RSS measurements without assistance from other information, e.g. IMU data. It is worth noting that, since the data were 


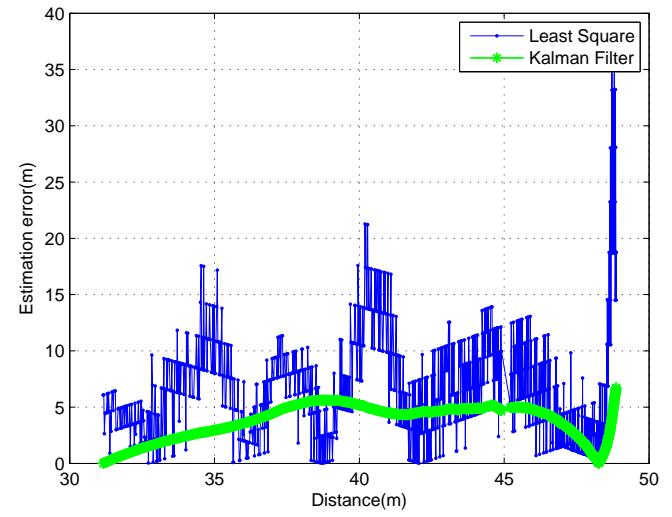

Fig. 9. Estimation of distance between transmitter and receiver from radio signal strength.

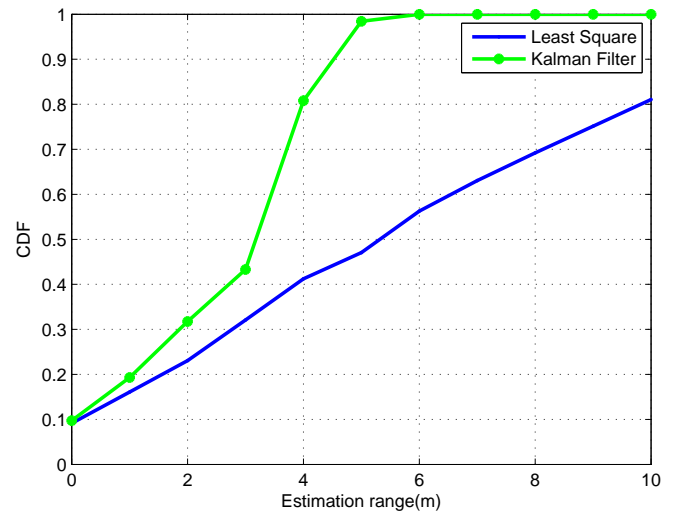

Fig. 10. The CDF of errors in distance estimation. The errors were calculated based on the estimated distance and its true value recorded by GPS.

collected at fairly short distances $(<60 \mathrm{~m})$, therefore the results obtained by using this dataset might not be able to reflect the measurements ideally under longer distances.

\section{B. Distance vs RSS}

The errors in estimation at different distances are shown in Fig.9. The RSS data measured from the experiments was used for estimation by the KF and LS algorithm respectively. The KF model is provided by (14) and (15) with an update rate of 10 times per second. From the figure, it is easy to see that the distance estimated by LS fluctuates severely during the movement of the UAV, thus it would produce large scale instant errors, which is confirmed by Fig.10. On the other hand, KF is more stable and produces estimates with higher accuracy as colored noise was considered in KF algorithm. From Fig.10, given $80 \%$ confidence, the estimation error of Kalman filters is about $4 m$, while that of LS method is about $10 \mathrm{~m}$. Generally, for both the two methods, increasing distance produces larger scale errors.

The difference in accuracy between these two approaches is mainly the result of how to exploit the historical data: LS uses current and historical data to model the system directly.
If there are large instantaneous discrepancies between the model and observation, the LS algorithm would produce large scale errors. However, for the KF algorithm, current observations only contribute a limited but necessary part (depending on the noise variance in measurement, see (19)), and the approach is thus more robust towards instantaneous changes. The accuracy of noise variance should be carefully handled in the KF algorithm.

In the following experiments, we study the effects of different noise type, path loss factor and IMU measurement (velocity) on the proposed algorithm in details. The dataset is the same as before.

1) Colored Noise vs White Noise: In this experiment, the performance of the white noise model and colored noise model are compared. Two experiments were conducted, where the first one assumed that the dataset has white noise and the second one assumed colored noise. The other parameters were the same for both the two cases.

Fig.11 (a) shows the performance comparison. From the figure, if KF only considers white noises, the error in distance estimation is much higher than that of the colored noise model. From the CDF figure, we can see that, given $80 \%$ confidence, the error of the colored noise model is about $4 \mathrm{~m}$ while that of the white noise algorithm is about $8.5 \mathrm{~m}$. It is worth mentioning that the difference between these two approach is not always the same, e.g. they are affected by the environment(e.g. noise variance).

2) Path Loss Factor $\gamma$ : In this experiment, we compared the impact of different $\gamma$ to the performance of distance estimation. A set of experiments involving adaptive $\gamma$ and fixed $\gamma$ were conducted by using the same dataset as before. Without losing generality, fixed $\gamma$ was chosen from the most common scope $[2,6]$.

The results are shown in Fig.11 (b). From the figure, it is interesting to notice that, within the defined range, the higher the path loss factor, the better the performance. The reason could be explained by multiple reflections and attenuations on the radio path. Therefore the path loss factor is likely to be highly unstable in a practical environment. The adaptive estimation algorithm proposed in this paper may not have the best performance all the time, but generally its errors in distance estimation are less than its counterparts with fixed values at the $70 \%$ of confidence and above.

3) With/Without IMU measurements: This experiment explicitly tested the effect of IMU information towards estimation accuracy in the proposed scheme. The Kalman filter (12) included velocity data integrated by the accelerometer, gyroscopic sensors and timer on the UAV. It is worth mentioning that in all of the previous experiments, IMU measurements were not used.

Fig.11 (c) shows the impact of using IMU information. The accuracy of estimation when IMU information is available is within $1.7 \mathrm{~m}$ given $80 \%$ of confidence, which is higher than the case without IMU data. The results suggest that if IMU data is available and accurate, it can be used in the proposed algorithm to improve the estimation accuracy. However, usually the accuracy of inertial sensors is time 
(a)

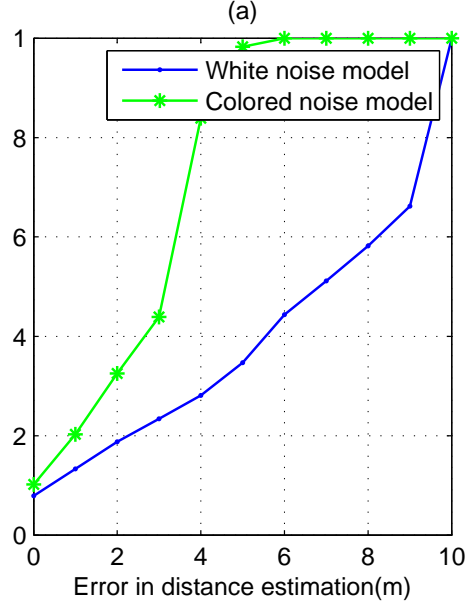

(b)

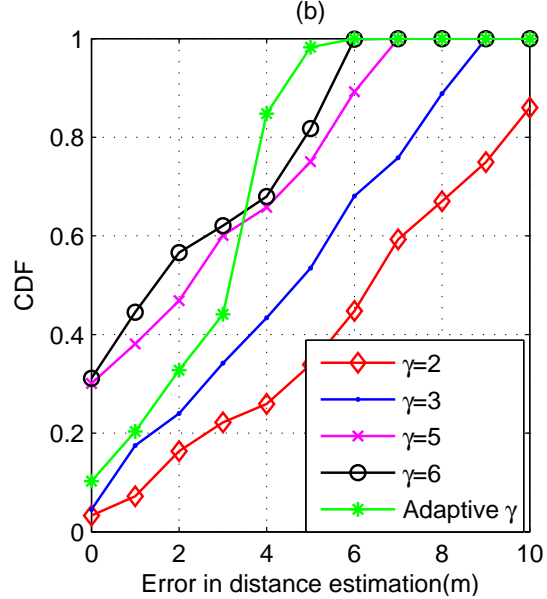

(c)

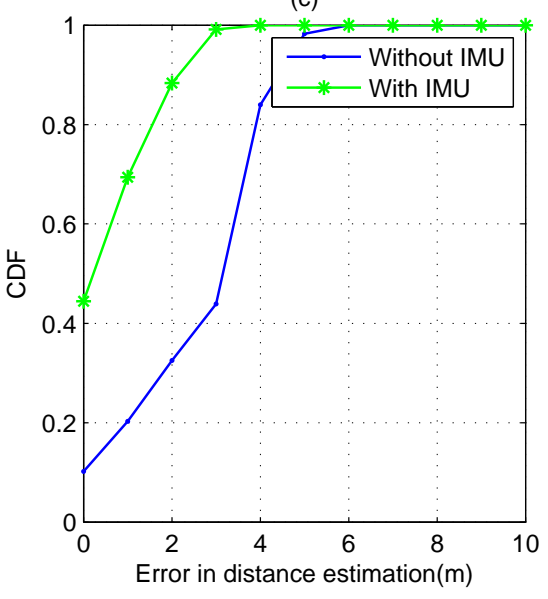

Fig. 11. Performance comparison given the same dataset. (a) The errors in estimation when using white noise model and colored noise model. (b) The errors in estimation when using fixed path loss factor and adaptive path loss factor. (c) The errors in estimation at the case with/without IMU information (velocity).

dependent - drifting with the increase of time, it is thus important to model and mitigate this effect [31].

\section{Safety Zone vs Collision}

In Section VI, a safety zone with radius $S$ is set for each UAV, where $S$ is given by (40) theoretically. In this experiment, given the accuracy of a single estimation (e.g. $80 \%$ of the errors in estimation are within $4 \mathrm{~m}$ or $16 \mathrm{~m}$ ), we examined the relationship between radius of safety zone and collision probability. 100, 000 independent trails of two UAVs were generated given two groups of estimation errors: $80 \%$ of errors were within $4 \mathrm{~m}$ or $16 \mathrm{~m}$. The maximum relative velocity was set to $10 \mathrm{~m} / \mathrm{s}$ and the UAVs always flew towards each other following this maximum speed. In this experiment, we only considered the collision events caused by imperfect estimation. The results are shown in Fig. 12.

From the figure, it is easy to see that under the same estimation error range, the radius of the safety zone has crucial effect on the collision probability of UAVs. The greater the radius, the lower the collision probability. The reason is that if the radius is large enough, multiple estimations can be carried out to provide higher overall accuracy. More importantly, the theoretical safety zone calculated from (40) matches the simulation results well. From the figure, given $80 \%$ of errors in estimation less than $4 m$, the probability of collision is $10^{-5}$ if the radius of safety zone is $115 \mathrm{~m}$. Similar probability values can also be found in the case of $16 \mathrm{~m}$ if the radius of safety zone is $230 \mathrm{~m}$. If we use (40) to calculate the radius of the safety zone, given the collision probability of $10^{-5}$, we can obtain the radius of the safety zone as $120 \mathrm{~m}$ in the first case and $240 \mathrm{~m}$ in the second case. In both cases, the calculated radius is sufficiently tight but also provides the required safety.

\section{Collision Detection and Avoidance}

In this experiment, the collision avoidance algorithm proposed in Section VII was tested. The scenario was

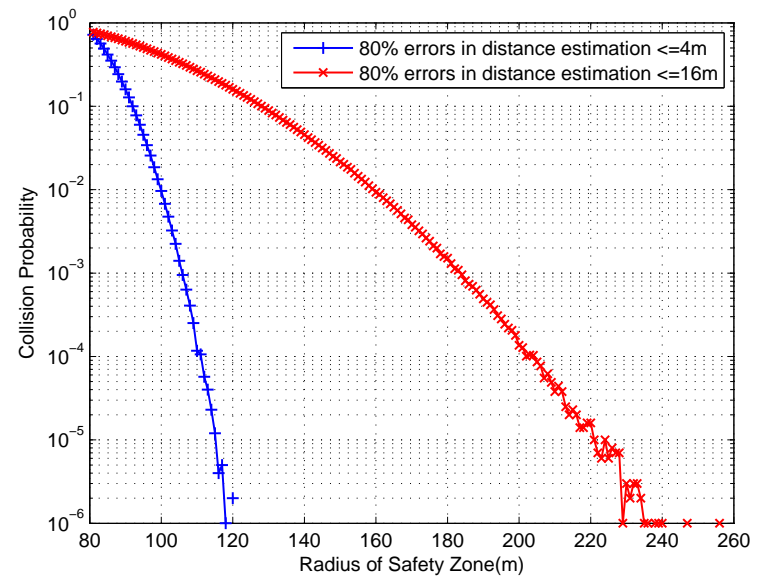

Fig. 12. The overall collision probability vs safety zone radius. $80 \%$ of errors in single estimation are within two ranges: $\leq 4 \mathrm{~m}$ and $\leq 16 \mathrm{~m}$. With the increase in safety zone, the collision probability decreases. At the same level of collision probability, larger estimation errors $(16 \mathrm{~m})$ need bigger safety zone.

set as follows: two UAVs working simultaneously, whose trajectories were generated randomly with estimation noise. These trajectories were very irregular, but should be much more smooth in practical situations where the trajectories are optimized. Nevertheless, they were good objects for the purpose of the test. The distance between the two UAVs was calculated given the imperfect position information. We set the radius of their safety zone as $120 \mathrm{~m}$, given the error in estimation as $4 \mathrm{~m}$ and the corresponding CDF as $80 \%$ from previous experiments. If the estimated distance was less than this radius, the trajectory modification algorithm proposed in VII would be called. Such a procedure was repeated until a safe trajectory was eventually obtained.

Fig.13 shows the trajectories of UAV1 and UAV2. We can see that for most of the time, their distance is less than the safety zone (See Fig.14). After applying the Orthogonal Rule, UAV2's trajectory is modified and the new distance is 


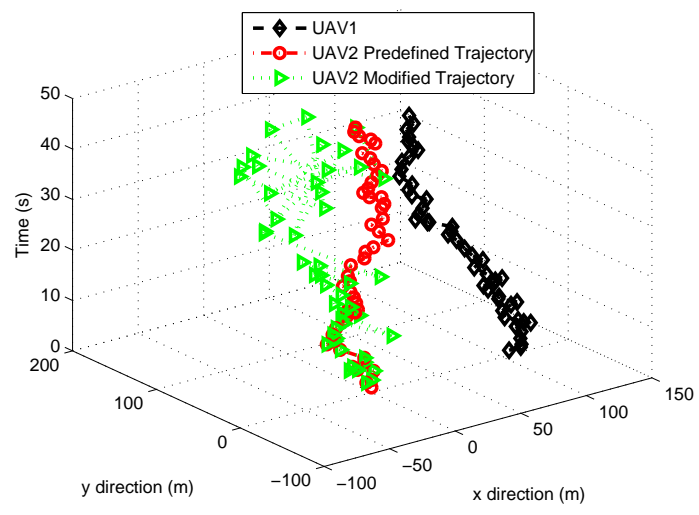

Fig. 13. The simulated trajectories of two UAVs. The trajectory of UAV2 is modified by following the Orthogonal Rule to avoid potential collisions. The new trajectory always respects the safety zone, but does not significantly divert from the predefined one. Distances between the two UAVs before and after modification are shown in Fig.14.

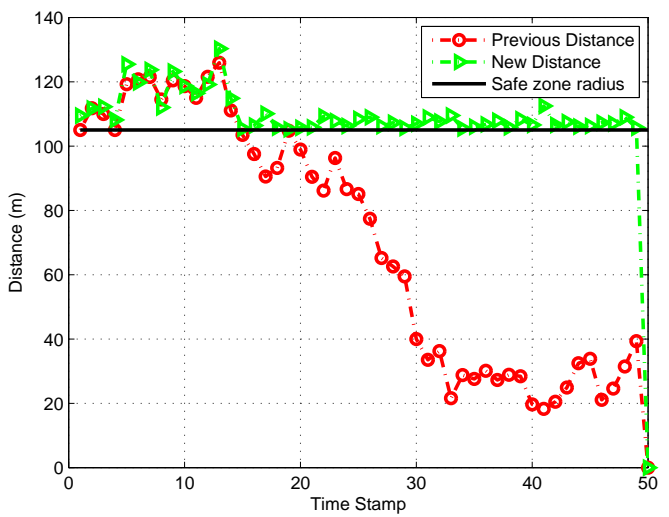

Fig. 14. Distances between the two UAVs before and after trajectory modification. The new distance should be greater than or equal to the radius of safety zone.

greater or equals to the safety zone radius, which confirms the effectiveness of the proposed trajectory modification rule.

\section{DisCUSSION}

\section{A. Number of Reference Nodes}

The following two cases should be considered. The first one is when there are more than three reference nodes, $J>3$. The three with the strongest RSS are chosen for the calculation of position. The reason of this choice is that, the ones with stronger signals are less affected by blocks and shadowing, thus the data estimated from their RSS is more accurate. The second case is when $J<3$. In this case the exact position of $U_{i}$ cannot be obtained. Only the distance (between $U_{i}$ and its neighbors) can be estimated. Even though such extreme case should be always avoided, when encountering this problem, based on the algorithm introduced in Section VI, the violation of the safety zone can still be detected using only the distance information.
Collision avoidance under this circumstance works as follows. The UAV with smaller ID should hover for one segment of time, recording the velocities of both two UAVs and distances. With the value of the two distances and direction of the other one's previous velocity, a triangle can be defined (The three vertexes of this triangle are the UAVs' old positions and the new position of the moving UAV.) and then a modified orthogonal rule can be used:

The Modified Orthogonal Rule: When two UAVs $\left(U_{i}, U_{j}, i<j\right)$ have potential collision, the velocity of the UAV with greater ID should be changed to be orthogonal to the current velocity of the other one, its value should be the same as the other UAVs and the direction of change should ensure the increase of distance.

Its implementation is as follows: The velocity $\mathbf{v}_{i}(n)$ of $U_{i}$ estimated by the inertial measurement unit (IMU) is transmitted to $U_{j}$. $U_{j}$ will change its movement following the modified orthogonal rule as above, where the details are similar to Section VII.

\section{B. Path Loss Factor $\gamma$}

In the free space model, the path loss factor is a constant, $\gamma=2$ [22]. However, its typical value in different indoor and outdoor environments and antenna heights is between 1.6 and 3.5. Office buildings with multiple floors may even have $\gamma$ as high as 6 . Higher frequencies tend to produce higher $\gamma$, which becomes lower at higher antenna heights [22], [32]. As a result, it is not accurate to pin a fixed number to a scenario. The experiments in Section VIII suggest that the path loss factor is sometimes a little higher than the typical values given in [22].

\section{Trajectory Modification for Collision Avoidance}

In this paper, we proposed to use the orthogonal rule to modify the trajectories of two UAVs when a potential collision is detected. This scheme is simple and effective, however, there might be better ways to find way points. For example, the movements of two UAVs can be explicitly compared and predicted to reduce potential conflicts. Furthermore, if the vehicles have high velocities, the way points found by the orthogonal rule should ideally be connected by smoothing trajectories rather straight lines. As stated in Section VII, genetic algorithms and higher order polynomial approximations can be used in this scenario. In the paper, we only study the control rule involving two UAVs. In real applications, more UAVs may have conflicts in the same space, thus a global collision avoidance algorithm will be proposed and tested in our future work.

\section{The Integration of Inertial Sensors}

The inertial sensors - accelerometer, gyros and magnetometers - are usually fused with the timer on the UAV to provide an estimate of the navigation states. For example, the accelerometers' orientation can be determined by the gyroscopic sensors. We can combine accelerometers' output and the time step of the system clock to obtain the 
UAV body velocity. Thus the position information can be obtained by integrating the velocities. Commercial inertial sensors should be calibrated using the data provided to adjust the bias and other determined errors. However, the accuracy of these sensors is time dependent - it degrades with the increase of time. Various methods have been studied to model the errors in an inertial sensor: [33], [34] proposed an autoregressivemoving-average model; [35], [36] used a stationary Gaussian-Markov process etc. It is worth noting that, if IMU data are integrated in the model (12), their time dependency should be compensated using algorithms, e.g. EKF filters [31].

\section{E. Limitations and Applications}

The proposed RSS based collision detection and avoidance scheme is targeted in open space where several UAVs are deployed. It is thus important to avoid collision if some of them do not have accurate position information. Indoor environments have much higher requirement on the accuracy and radius of safety zones. Furthermore, the concrete walls and indoor objects have strong degradation and reflection towards the RF signal, it is thus very difficult to use the RF signal alone in collision avoidance. In this case, we have combined several techniques, e.g. RSS with wireless beacons, ultrasonic and optical sensors etc., to provide the required accuracy. It is also an interesting topic for further research.

\section{Conclusion}

This paper studies the application of radio frequency signal to UAV position estimation and collision avoidance. The radio signal model and its noise component are analyzed. The influence of colored noise and path loss factor are carefully handled. The Kalman filter algorithm is then proposed to estimate the distance between receiver and transmitter. The collision probability of using RSS is studied and a collision avoidance strategy is also proposed with verification of its effectiveness by experiments. The proposed algorithms can be used for UAVs which are cooperatively deployed for sensing tasks and other missions.

\section{APPENDIX A}

\section{The PROOF OF THEOREM 1}

Proof: The left hand side of (27) can be expanded using Taylor series, given by (The iteration index $s$ is ignored here for the benefit of concise expression.)

$$
\begin{array}{r}
\varepsilon^{\mathrm{T}}(\gamma+\theta \Theta) \varepsilon(\gamma+\theta \Theta)-\varepsilon^{\mathrm{T}}(\gamma) \varepsilon(\gamma) \\
=\frac{\partial \varepsilon^{\mathrm{T}}(\gamma) \varepsilon(\gamma)}{\partial \gamma} \Theta \theta+o \theta^{2} .
\end{array}
$$

Since

$$
\begin{aligned}
\frac{\partial \varepsilon^{\mathrm{T}}(\gamma) \varepsilon(\gamma)}{\partial \gamma} \Theta & =-2 \varepsilon^{\mathrm{T}}(\gamma) \frac{\partial \mathbf{F}}{\partial \gamma} \Theta \\
& =-2(\mathbf{L}-\mathbf{F}(\gamma))^{\mathrm{T}} J(\gamma) \Theta \\
& =-2 \| \mathbf{Z}_{J}\left(\mathbf{L}-\mathbf{F}(\gamma) \|^{2}\right.
\end{aligned}
$$

where $\mathbf{Z}_{J}=J(\gamma) J^{-1}(\gamma)$. Replace the corresponding part of (46) and arrange the equation gives

$$
\begin{aligned}
& \varepsilon^{\mathrm{T}}(\gamma+\theta \Theta) \varepsilon(\gamma+\theta \Theta)-\varepsilon^{\mathrm{T}}(\gamma) \varepsilon(\gamma) \\
& \quad=\left(-2 \| \mathbf{Z}_{J}\left(\mathbf{L}-\mathbf{F}(\gamma) \|^{2}+o \theta\right) \theta\right.
\end{aligned}
$$

From the features of Taylor series, we know that $o \theta$ has equal or higher order than $\theta$. When $\theta \rightarrow 0$, it is easy to know $o \rightarrow 0$. As a result,

$$
\varepsilon^{\mathrm{T}}(\gamma+\theta \Theta) \varepsilon(\gamma+\theta \Theta)-\varepsilon^{\mathrm{T}}(\gamma) \varepsilon(\gamma)<0 .
$$

Thus the theorem is proved.

\section{ACKNOWLEDGMENT}

This work is funded by the EPSRC SUAAVE (Sensing Unmanned Autonomous Aerial Vehicles) project (grant number: EP/F064179/1). The authors would like to thank the anonymous reviewers for their helpful comments and suggestions on the collision detection and avoidance algorithms, and Simon J. Julier for the discussion about coloured noise.

\section{REFERENCES}

[1] T. Lam, H. Boschloo, M. Mulder, and M. van Paassen, "Artificial force field for haptic feedback in uav teleoperation," IEEE Trans. Syst., Man, Cybern. A, Syst. ,Humans, vol. 39, no. 6, pp. 1316 1330, Nov. 2009.

[2] I. Akyildiz and M. Vuran, Wireless Sensor Networks, ser. Ian F. Akyildiz Series in Communications and Networking. Wiley, 2010

[3] W. Teacy, J. Nie, S. McClean, and G. Parr, "Maintaining connectivity in uav swarm sensing," in GLOBECOM Workshops (GC Wkshps), 2010 IEEE, Dec. 2010, pp. $1771-1776$.

[4] C. Luo, P. Ward, S. Cameron, G. Parr, and S. McClean, "Uav position estimation and collision avoidance using the extended kalman filter," in GLOBECOM Workshops (GC Wkshps), 2012 IEEE, Dec. 2012.

[5] S. Cameron, S. Hailes, S. McClean, and et al., "Suaave: Combining aerial robots and wireless networking," in SUAAVE. University of Oxford, University College London, University of Ulster, Feb. 2010, pp. $1-14$.

[6] X. Wang, V. Yadav, and S. Balakrishnan, "Cooperative uav formation flying with obstacle/collision avoidance," IEEE Trans. Control Syst. Technol., vol. 15, no. 4, pp. 672 -679, July 2007.

[7] P. Huang and Y. Pi, "Urban environment solutions to gps signal nearfar effect,” IEEE Aerosp. Electron. Syst. Mag., vol. 26, no. 5, pp. 18 -27 , May 2011.

[8] M. Bryson and S. Sukkarieh, "Observability analysis and active control for airborne slam," IEEE Trans. Aerosp. Electron. Syst., vol. 44, no. 1, pp. $261-280$, Jan. 2008.

[9] X. Chai and Q. Yang, "Reducing the calibration effort for probabilistic indoor location estimation," IEEE Trans. Mobile Comput., vol. 6, no. 6, pp. $649-662$, June 2007.

[10] S. Golden and S. Bateman, "Sensor measurements for wi-fi location with emphasis on time-of-arrival ranging," IEEE Trans. Mobile Comput., vol. 6, no. 10, pp. $1185-1198$, Oct. 2007

[11] N. N. Patwari, N. Patwari, A. O. H. Iii, M. Perkins, N. S. Correal, and R. J. Odea, "Relative location estimation in wireless sensor," IEEE Trans. Signal Process., vol. 51, pp. 2137-2148, 2003.

[12] J. M.Sather and A. MacDonald, "Mobile-assisted network location system (mnls) overview," in Wireless E911 Phase IIWaiver Request Filed by AT\&T Wireless Services. FCC docket, Apr. 2001, pp. 94 -102 .

[13] A. Weiss, "On the accuracy of a cellular location system based on rss measurements," IEEE Trans. Veh. Technol., vol. 52, no. 6, pp. 1508 - 1518, Nov. 2003

[14] D. Arora and M. McGuire, "Lower bounds on mobile terminal localisation in an urban area," Communications, IET, vol. 5, no. 9, pp. $1182-1191$, June 2011.

[15] X. Li, "Rss-based location estimation with unknown pathloss model," IEEE Trans. Wireless Commun., vol. 5, no. 12, pp. 3626 -3633 , Dec. 2006. 
[16] T. Roos, P. Myllymaki, and H. Tirri, "A statistical modeling approach to location estimation," IEEE Trans. Mobile Comput., vol. 1, no. 1, pp. 59 - 69, Jan. - Mar. 2002.

[17] H. Laitinen, S. Juurakko, T. Lahti, R. Korhonen, and J. Lahteenmaki, "Experimental evaluation of location methods based on signalstrength measurements," IEEE Trans. Veh. Technol., vol. 56, no. 1, pp. $287-296$, Jan. 2007.

[18] A. S. C. Technical Staff and A. Gelb, Applied optimal estimation. MIT Press, 1999.

[19] J. D. Parsons, The Mobile Radio Propagation Channel, 2nd Edition, 2nd ed. Wiley, 2000.

[20] L. C. Ng, "On the application of allan variance method for ring laser gyro performance characterization," Oct., p. 29, 1993.

[21] S. Bednarz and P. Misra, "Receiver clock-based integrity monitoring for gps precision approaches," IEEE Trans. Aerosp. Electron. Syst., vol. 42 , no. 2, pp. $636-643$, Apr. 2006

[22] A. Goldsmith, Wireless communications. Cambridge University Press, 2005

[23] D. Allan, "Statistics of atomic frequency standards," Proceedings of the IEEE, vol. 54, no. 2, pp. 221 - 230, Feb. 1966

[24] M. Tehrani, "Ring laser gyro data analysis with cluster sampling technique," in Proc. SPIE, vol. 412, 1983, pp. 207-222.

[25] A. Bryson and D. Johansen, "Linear filtering for time-varying systems using measurements containing colored noise," IEEE Trans. Autom. Control, vol. 10, no. 1, pp. 4 - 10, Jan. 1965.

[26] R. G. Brown and P. Y. C. Hwang, Introduction to Random Signals and Applied Kalman Filtering, P. Y. C. Hwang, Ed. John Wiley \& Sons, 1997, vol. 2, no. 4

[27] C. Tomlin, G. J. Pappas, and S. Sastry, "Conflict resolution for air traffic management: a study in multiagent hybrid systems," IEEE Trans. Autom. Control, vol. 43, no. 4, pp. 509-521, 1998.

[28] G. Bianchi, "Performance analysis of the ieee 802.11 distributed coordination function," IEEE J. Sel. Areas Commun., vol. 18, no. 3, pp. 535-547, 2000 .

[29] J. Rossiter, Model-based predictive control: a practical approach, ser. CRC Press control series. CRC Press, 2003

[30] D. Dimarogonas, "Sufficient conditions for decentralized potential functions based controllers using canonical vector fields," IEEE Trans. Autom. Control, vol. 57, no. 10, pp. 2621 -2626, Oct. 2012.

[31] B. Barshan and H. F. Durrant-Whyte, "Inertial navigation systems for mobile robots," IEEE Trans. Robot. Autom., vol. 11, no. 3, pp. 328 - 342, June 1995.

[32] V. Erceg, L. Greenstein, S. Tjandra, S. Parkoff, A. Gupta, B. Kulic, A. Julius, and R. Bianchi, "An empirically based path loss model for wireless channels in suburban environments," IEEE J. Sel. Areas Commun., vol. 17, no. 7, pp. $1205-1211$, jul 1999.

[33] A. S. Oravetz and H. J. Sandberg, "Stationary and nonstationary characteristics of gyro drift rate," AIAA Journal, vol. 8, pp. 17661772,1970

[34] S. Paniit and W. Zhang, "Modeling random gyro drift rate by data dependent systems," IEEE Trans. Aerosp. Electron. Syst., vol. AES22, no. 4, pp. 455 -460, July 1986.

[35] R. L. Hammon, "An application of random process theory to gyro drift analysis," Aeronautical and Navigational Electronics, IRE Transactions on, vol. ANE-7, no. 3, pp. 84 -91, Sept. 1960.

[36] B. Danik, "The least-square approximation of inertial platform drift," IEEE Trans. Aerosp. Electron. Syst., vol. AES-2, no. 5, pp. 591 -594, Sept. 1966.

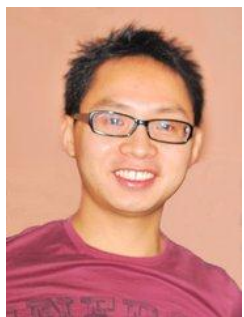

Chunbo Luo received the B.Eng. and M.Eng degrees in Electronic Engineering from the University of Electronic Science and Technology of China (UESTC), Chengdu, China, in 2005 and 2007, respectively, and the Ph.D. degree from the University of Reading, UK in 2011. $\mathrm{He}$ is currently working on the EPSRC SUAAVE project at the University of Ulster. His research interests include wireless networks and signal processing, at the moment, he is particularly interested in wireless sensor networks and their applications on robot and UAV platforms.

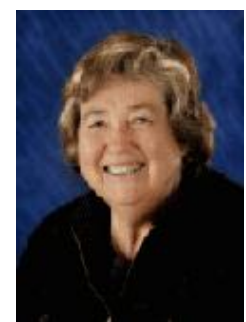

Sally I. McClean is Professor of Mathematics and Leader of the Information and Communications Engineering Research Group at the University of Ulster. She joined the University of Ulster after receiving a MA in mathematics from Oxford University, a M.Sc. in mathematical statistics and operational research from Cardiff University and $\mathrm{PhD}$ in Mathematics (stochastic modelling) from the University of Ulster, She has over 250 publications, with research interests in mathematical modelling, applied probability, multivariate statistical analysis, and applications of mathematical and statistical methods to computer science, particularly database, telecommunications and sensor technology. She is Fellow of the Royal Statistical Society, Fellow of the Operational Research Society, Associate Fellow of the Institute of Mathematics and its Applications and Member of the IEEE. In addition she is past president and current treasurer of the Irish Statistical Association.

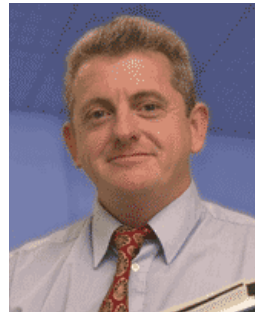

Gerard Parr holds the Full Chair in Telecommunications Engineering and is a Member of the Computer Science Research Institute in the Faculty of Computing \& Engineering at the University of Ulster in Coleraine, Northern Ireland He holds a Ph.D. in Self Stabilizing Protocols, aspects of which were completed with one of the founding Fathers of the Internet (Professor Jon Postel) as a Visiting Research Scientist at the USC Information Sciences Institute in Marina Del Rey, Los Angeles on US-DARPA funded research projects into Fault-Tolerant and Self-stabilizing Protocols. Areas of research within the Ulster group include Wireless Sensor Clouds, Disaster Response Communications, event suppression for wireless sensor devices, delay-sensitive protocols, and energy-aware autonomic networking for sensor-clouds and edge computing. He has attracted several millions of external research and commercial funding and has advised governments on the allocation of funding to projects valued in total of approximately 4.5 billion. Professor Parr is a member of the UK Engineering and Physical Sciences Research Council (EPSRC) Expert Peer Review College.

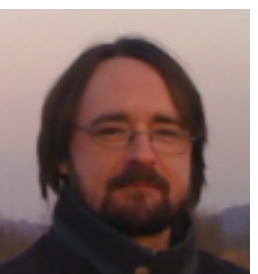

Luke Teacy is a Senior Research Fellow in the Agents, Complexity and Interaction Group at the University of Southampton, UK. His research interests include coordination and planning under uncertainty in multiagent systems, and Bayesian methods for online learning and sequential decision making. At the University of Ulster, he was previously employed on the EPSRC SUAAVE project, on which he developed learning and planning techniques for coordinating the actions multiple UAVs. Luke is currently involved with the EPSRC MOSAIC project, aiming to develop multiagent control and planning techniques for future applications of autonomous air and ground vehicles.

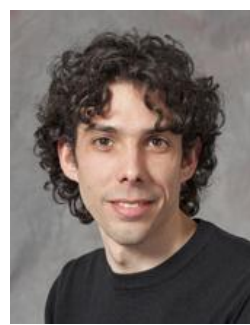

Renzo De Nardi received the M.S. degree in telecommunications from The University of Padua, Italy in 2004 and the Ph.D. in computer science from the University of Essex, UK in 2010. Since 2009 he is Research Associate at the Department of Computer Science, University College London. His research interest lies in the areas of modelling, control and learning with application to aerial vehicles. 